\title{
Salmonella Heidelberg Strain Responses to Essential Oil Components
}

\author{
Juliany Rivera Calo ${ }^{1}$, Christopher A. Baker ${ }^{1}$, Si Hong Park ${ }^{1} \&$ Steven C. Ricke ${ }^{1}$ \\ ${ }^{1}$ Center for Food Safety; Department of Food Science, 2650 N. Young Ave., University of Arkansas, \\ Fayetteville, AR 72704, USA \\ Correspondence: Steven C. Ricke, Center for Food Safety; Department of Food Science, University of Arkansas, \\ Fayetteville, AR 72704, USA. Tel: 1-479-575-4678. E-mail: sricke@uark.edu
}

\author{
Received: May 5, 2015 Accepted: June 28, 2015 Online Published: August 13, 2015 \\ doi:10.5539/jfr.v4n5p73 URL: http://dx.doi.org/10.5539/jfr.v4n5p73
}

\begin{abstract}
Salmonella are one of the more prominent foodborne pathogens that represent a major health risk to humans. Salmonella serovar Heidelberg strains are increasingly becoming an important public health concern, since they have been identified as one of the primary Salmonella serovars responsible for human outbreaks. Over the years, Salmonella Heidelberg isolates have exhibited higher rates of resistance to multiple antimicrobial agents compared to other Salmonella serovars. Essential oils (EOs) have been widely used as alternatives to chemical-based antimicrobials. In the current research, five EOs were screened to determine their antimicrobial activity against $15 S$. Heidelberg strains from different sources. Oils tested were $\mathrm{R}(+)$-limonene, orange terpenes, cold compressed orange oil, trans-cinnamaldehyde and carvacrol. EOs were stabilized in nutrient broth by adding $0.15 \%(\mathrm{w} / \mathrm{v})$ agar. Tube dilution assays and minimal inhibitory concentrations (MIC) were determined by observing color changes in samples during exposure to EOs. Carvacrol and trans-cinnamaldehyde completely inhibited the growth of $S$. Heidelberg strains, while $\mathrm{R}(+)$-limonene and orange terpenes did not show any inhibitory activity against the strains tested. Cold compressed orange oil only inhibited growth of two of the strains exhibiting an MIC of $1 \%$. All $S$. Heidelberg isolates evaluated exhibited similar responses to the respective EOs. The use of all natural antimicrobials such as specific EOs offers the potential to limit the majority of $S$. Heidelberg isolates that may occur in food production.
\end{abstract}

Keywords: essential oils, Salmonella Heidelberg; antimicrobials

\section{Introduction}

Foodborne illnesses continue to be one of the primary public health concerns in the United States (U. S.), and it has been estimated that over 1 million Americans contract Salmonella each year (Scallan et al., 2011). Annual costs for Salmonella control efforts are estimated to be $\$ 14.6$ billion (Scharff, 2010; Heithoff et al., 2012). Salmonella is not only a public health concern due to the number of cases per year, but many strains have developed resistance to antimicrobial agents (Kim et al., 2005; Foley \& Lynne, 2008; Bajpai, Baek, \& Kang, 2012) due to continued therapeutic use of antimicrobials in feed products ( $\mathrm{Su}$, Chiu, $\mathrm{Chu}$, \& Ou, 2004; Kim et al., 2005).

Salmonella enterica serovar Heidelberg ( $S$. Heidelberg) ranks fourth among the top five serovars associated with human infections and is responsible for an estimated 84,000 illnesses in the U. S. annually (CDC, 2008; FDA, 2009; Foley et al., 2011; Han et al., 2011). Salmonella Heidelberg is one of the most commonly isolated serovars in the U.S. and Canada from clinical cases of salmonellosis, retail meats and livestock (Zhao et al., 2008; Hur, Jawale, \& Lee, 2012). While most Salmonella infections are self-limiting and are resolved within a few days, $S$. Heidelberg tends to cause a significantly higher percentage of invasive infections (Vugia et al., 2004; Han et al., 2011). As a result, antimicrobial therapy is often necessary, making antimicrobial resistance a significant concern. Due to the tendency of $S$. Heidelberg to cause severe extra-intestinal infections (Wilmshurst \& Sutcliffe, 1995) such as myocarditis and septicemia (Vugia et al., 2004), the occurrence of $S$. Heidelberg multidrug resistance strains is of extreme clinical importance. Salmonella Heidelberg strains exhibiting antimicrobial resistance have been isolated from humans, retail meats and food animals (Logue, Sherwood, Olah, Elijah, \& Dockter, 2003; Nayak et al., 2004; Kaldhone et al., 2008; Zhao et al., 2008; Lynne, Kaldhone, David, White, \& Foley, 2009; Oloya, Doetkott, \& Khaitsa, 2009; Han et al., 2011). Studies suggest that poultry-associated $S$. Heidelberg strains harbor IncFIB, IncA/C, IncH2, and IncI1 plasmids, which may contain genes that confer resistance to several antibiotics such as tetracycline, kanamycin, streptomycin, and sulfonamides (Han et al., 2012). Because $S$. 
Heidelberg is responsible for causing more invasive infections compared to other serovars, it is important to monitor its prevalence and resistance - novel and unique intervention strategies are a priority to reduce or eliminate its presence.

Aromatic plants and their extracts have been examined for their effectiveness in food safety and preservation applications (Fisher \& Phillips, 2008). Essential oils, for example, exhibit antimicrobial properties that may make them suitable alternatives to antibiotics (Chaves et al., 2008). These potential attributes and an increasing demand for natural medicinal treatment options have brought attention to the use of EOs as potential antimicrobial alternatives (Fisher \& Phillips, 2008; Solórzano-Santos \& Miranda-Novales, 2012; Rivera Calo, Crandall, O’Bryan, \& Ricke, 2015a). Essential oils derived as by-products in citrus processing have been screened for antimicrobial properties against common foodborne pathogens, and several of these compounds have exhibited antimicrobial properties (Dabbah, Edwards, \& Moats, 1970; Rivera Calo et al., 2015a).

Alali et al. (2013) evaluated the use and application of various EOs in broiler drinking water to reduce ceca colonization and fecal shedding of $S$. Heidelberg. Although the EOs evaluated were not a sufficient means to limit $S$. Heidelberg isolates in ceca or reduce shedding, these authors reported a reduction of $S$. Heidelberg colonization in the crops of seeders as well as an increased weight gain in birds that were administered the EOs (Alali et al., 2013). This research was conducted to determine the efficacy of several EOs on $S$. Heidelberg isolates that originated from different sources.

\section{Materials and Methods}

\subsection{Bacterial Strains Used in This Study}

Fifteen different $S$. Heidelberg strains were evaluated (Table 1). Strains were isolated from multiple sources such as, turkey and ground turkey, poultry products, cattle, swine, poultry and poultry egg houses, and humans.

Table 1. Salmonella Heidelberg strains used in this study

\begin{tabular}{lll}
\hline Strain & Source & Reference \\
\hline ARI-14 & poultry products & In this study \\
SL 476 & ground turkey & Fricke et al., 2011 \\
SL 486 & human & Fricke et al., 2011 \\
692 & chicken egg house & Lynne et al., 2009 \\
945 & human & Han et al., 2011 \\
114 & cattle & Lynne et al., 2009 \\
163 & turkey & Kaldhone et al., 2008 \\
136 & swine & Lynne et al., 2009 \\
1148 & human & Han et al., 2012 \\
824 & turkey & Kaldhone et al., 2008 \\
130 & chicken & Lynne et al., 2009 \\
937 & human & Han et al., 2011 \\
118 & cattle & Lynne et al., 2009 \\
144 & swine & Lynne et al., 2009 \\
146 & swine & Lynne et al., 2009 \\
\hline
\end{tabular}

\subsection{DNA Extraction}

One colony of each $S$. Heidelberg strain was inoculated in $5 \mathrm{~mL}$ Luria Bertani (LB) broth and incubated at $37^{\circ} \mathrm{C}$, $190 \mathrm{rpm}$ for $16 \mathrm{~h}$ in a C76 Water Bath Shaker (New Brunswick Scientific, Edison, NJ, USA). A one mL aliquot of bacterial cells was transferred to a microcentrifuge tube, and samples were centrifuged at $14,000 \times g$ for 10 min and the corresponding supernatants were discarded. Genomic DNA was extracted with the Qiagen DNeasy Blood Tissue kit (Qiagen, Valencia, CA, USA) according to the manufacturer's instruction. The isolated genomic DNA concentration and purity were measured using a NanoDrop ND-1000 (Thermo Scientific, Wilmington, DE, 
USA) and DNA samples were subsequently stored at $-20^{\circ} \mathrm{C}$.

\subsection{S. Heidelberg Confirmation by PCR}

A conventional PCR assay was conducted with a MJ Mini Personal Thermal Cycler (Bio-Rad, Hercules, CA, USA). Salmonella Heidelberg specific primers were generated based on the type II restriction enzyme methylase subunit sequences of $S$. Heidelberg (Bronowski \& Winstanley, 2009), and upon evaluation of the specificity for all 15 strains, each was determined to belong to the serovar Heidelberg (Table 2). Additionally, exclusivity assays were performed on different Salmonella serovars as well as other non-Salmonella. The total reaction (25 $\mu \mathrm{L}$ volume) was composed of $1 \mu \mathrm{L}$ of template DNA, $1 \mu \mathrm{L}$ of each primer (IDT, Coralville, IA, USA), $12.5 \mu \mathrm{L}$ of SYBR Green (Cambrex Bioscience, Walkersville, MD, USA), and $9.5 \mu \mathrm{L}$ of DNase-RNase free water. The PCR conditions consisted of pre-denaturation at $94{ }^{\circ} \mathrm{C}$ for $5 \mathrm{~min}, 35$ cycles of denaturation at $94{ }^{\circ} \mathrm{C}$ for $30 \mathrm{~s}$, annealing at $65{ }^{\circ} \mathrm{C}$ for $30 \mathrm{~s}$, extension at $72{ }^{\circ} \mathrm{C}$ for $30 \mathrm{~s}$, and a final extension cycle at $72{ }^{\circ} \mathrm{C}$ for $5 \mathrm{~min}$. PCR products were confirmed on a $1.5 \%$ agarose gel and visualized on a transilluminator (Bio-Rad, Hercules).

Table 2. Salmonella Heidelberg specific primer pair used in this study

\begin{tabular}{llrl}
\hline Primer & Sequence & Product & Reference \\
\hline SH_SHP-2f & 5'-GCATA GTTCC AAAGC ACGTT-3' & 180 & This study \\
SH_SHP-1r & 5'-GCTCA ACATA AGGGA AGCAA-3' & 180 & This study \\
\hline
\end{tabular}

\subsection{Essential Oils and Cultures}

Each of the EOs evaluated $(\mathrm{R}(+)$-limonene, orange terpenes, cold compressed orange oil, trans-cinnamaldehyde and carvacrol) were obtained from Sigma Aldrich (St. Louis, MO, USA). Fifteen $S$. Heidelberg strains from different sources were evaluated (Table 1). One colony of each strain was inoculated into Luria-Bertani (LB) broth and incubated at $42{ }^{\circ} \mathrm{C}, 190 \mathrm{rpm}$ for $18 \mathrm{~h}$. In addition, analyses were performed on $S$. Heidelberg strains incubated at $37^{\circ} \mathrm{C}, 190 \mathrm{rpm}$ for $18 \mathrm{~h}$.

\subsection{Modified Tube Dilution Assay}

To maintain the EOs in a homogeneous mixture, $0.15 \%$ agar was added to nutrient broth (NB), boiled for 1 min and subsequently autoclaved. Once the media cooled to room temperature, triphenyl tetrazolium chloride (TTC) $(1 \%)$ was added to act as a growth indicator. As previously described by O'Bryan et al. (2008), serial dilutions were made by placing $10 \mathrm{~mL}$ of the $\mathrm{NB}$ with $0.15 \%$ agar (NBA) in the first tube and $5 \mathrm{~mL}$ in the remaining tubes for a total of four tubes. One hundred $\mu \mathrm{L}$ of the essential oil was added to the $1^{\text {st }}(10 \mathrm{~mL})$ tube for an initial $1 \%$ concentration. To perform serial dilutions, $5 \mathrm{~mL}$ of the emulsion was transferred to the next tube, and the procedure was repeated for a total of four dilutions. Five $\mathrm{mL}$ were removed from the last tube and discarded so that all tubes consisted of equal volumes $(5 \mathrm{~mL})$. All tubes were inoculated with $50 \mu \mathrm{L}$ of an overnight culture of each $S$. Heidelberg strain, and the tubes were subsequently incubated for $24 \mathrm{~h}$ at $37^{\circ} \mathrm{C}$. A change in color from light yellow to pink/red indicated growth. Minimum inhibitory concentrations were determined as the lowest concentration of EOs that showed no color change in the medium, which was considered representative of the absence of detectable bacterial growth.

\section{Statistical Analysis}

MIC tests were repeated as three independent trials. Each mean \pm standard deviation was determined using JMP Pro Software Version 11.0 (SAS Institute Inc., Cary, NC).

\section{Results and Discussion}

Salmonella Heidelberg specific primers (Table 2) were used to confirm that all 15 strains evaluated belong to $S$. Heidelberg (Figure 1). Park and Ricke (2015) developed a multiplex PCR assay to simultaneously detect Salmonella serovars Heidelberg, Enteriditis, and Typhimurium. The $S$. Heidelberg primers were generated for this study similar to Park and Ricke (2015), which involves BLAST alignment(s) and subsequent inclusivity and exclusivity assays for primer specificity confirmation. In addition to the potential detection applications, the $S$. Heidelberg specific primers used in this study suggest that although these isolates originated from different environments, there is a certain level of genetic uniformity among these $S$. Heidelberg isolates.

Minimum inhibitory concentrations were determined by observing tubes for any color change to pink or red (Table 3). For the $S$. Heidelberg strains grown at $42{ }^{\circ} \mathrm{C}$, results indicate that trans-cinnamaldehyde and carvacrol 
exhibited MICs of $131 \mu \mathrm{g} / \mathrm{mL}$ and $122 \mu \mathrm{g} / \mathrm{mL}$, respectively, by completely inhibiting the growth of each $S$. Heidelberg strain, while orange terpenes and $\mathrm{R}(+)$-limonene did not exhibit any inhibitory activity against any of these strains. Cold compressed orange oil only inhibited growth of two of the strains (945 and 114), with MIC's of $843 \mu \mathrm{g} / \mathrm{mL}$. No relationship was observed between the source of the strain and the EOs tested. Similar results were obtained when strains were grown at $37{ }^{\circ} \mathrm{C}$, although under this condition cold compressed orange oil showed no antimicrobial activity for all strains, while at $42{ }^{\circ} \mathrm{C}$ it inhibited strains 945 and 114 with an MIC of $843 \mu \mathrm{g} / \mathrm{mL}$.

Table 3. Minimum inhibitory concentrations $(\mu \mathrm{g} / \mathrm{mL})$ of five EOs against Salmonella Heidelberg isolates

\begin{tabular}{llllll}
\hline Isolate & \multicolumn{2}{l}{ Essential oils } & & \\
& carvacrol & $\mathrm{R}(+)$-limonene & orange terpenes & orange oil & trans-cinnamaldehyde \\
\hline SL 486 & $122 \pm 0$ & No effect & No effect & No effect & $131 \pm 0$ \\
SL 476 & $122 \pm 0$ & No effect & No effect & No effect & $131 \pm 0$ \\
ARI-14 & $122 \pm 0$ & No effect & No effect & No effect & $131 \pm 0$ \\
692 & $122 \pm 0$ & No effect & No effect & No effect & $131 \pm 0$ \\
945 & $122 \pm 0$ & No effect & No effect & $843 \pm 0$ & $131 \pm 0$ \\
114 & $122 \pm 0$ & No effect & No effect & $843 \pm 0$ & $131 \pm 0$ \\
163 & $122 \pm 0$ & No effect & No effect & No effect & $131 \pm 0$ \\
136 & $122 \pm 0$ & No effect & No effect & No effect & $131 \pm 0$ \\
1148 & $122 \pm 0$ & No effect & No effect & No effect & $131 \pm 0$ \\
824 & $122 \pm 0$ & No effect & No effect & No effect & $131 \pm 0$ \\
130 & $122 \pm 0$ & No effect & No effect & No effect & $131 \pm 0$ \\
937 & $122 \pm 0$ & No effect & No effect & No effect & $131 \pm 0$ \\
118 & $122 \pm 0$ & No effect & No effect & No effect & $131 \pm 0$ \\
144 & $122 \pm 0$ & No effect & No effect & No effect & $131 \pm 0$ \\
146 & $122 \pm 0$ & No effect & No effect & No effect & $131 \pm 0$ \\
\hline
\end{tabular}

There are several indications that there may be differences in responses among Salmonella serovars and in some cases even among strains. This is critical to consider since overall recommendations for antimicrobial application to Salmonella can sometimes be made based on the responses of only a few serovars and/or strains. The growth responses of $S$. Typhimurium grown in spent media generated from several of the $S$. Heidelberg isolates used in this study have been evaluated (Rivera Calo, Park, Baker, \& Ricke, 2015b). The spent media from twelve of the fifteen $S$. Heidelberg isolates evaluated in this study decreased the growth of $S$. Typhimurium (ATCC 14028), which suggests that $S$. Heidelberg isolates may compete in some manner with other Salmonella serovars (Rivera Calo et al., 2015b). Additionally, studies have shown the existence of differences between serovars as well as strains within the same serovar of Salmonella enterica can occur for certain antimicrobials. In their research, González et al. (2012) compared hilA gene expression in response to acid stress between different Salmonella serovars and strains. Results showed that there are serovar and strain differences in virulence gene expression and acid tolerance; regulation of hilA showed to be serovar and strain dependent as well as dependent on acid type (González et al., 2012). In an extensive genome analysis comparison between UK-1 and other $S$. Typhimurium strains, Luo et al. (2012) reported that virulence factors pertaining to one strain might increase or decrease virulence when present in a different strain. Shah et al. (2011) reported that isolates from $S$. Enteritidis that have been recovered from poultry or poultry environment are not equally pathogenic, nor do they have similar invasiveness.

Previous research has shown that different EOs exhibit antimicrobial activity pre- and post-harvest on Salmonella. The antimicrobial effect of EOs has been reported to be concentration dependent (Sivropoulou et al. 1996). Ravishankar et al. (2010) reported that at a $0.2 \%$ concentration, antimicrobials, carvacrol and cinnamaldehyde completely inactivated the antibiotic-resistant and -susceptible isolates of $S$. enterica. Zhou et al. (2007) investigated the antimicrobial activity of cinnamaldehyde, thymol and carvacrol individually and in 
combination against $S$. Typhimurium, and reported the lowest concentrations of cinnamaldehyde, thymol and carvacrol inhibiting the growth of $S$. Typhimurium significantly were 200,400 and $400 \mathrm{mg} / \mathrm{L}$, respectively. When combined, cinnamaldehyde/thymol, cinnamaldehyde/carvacrol and thymol/carvacrol demonstrated that the concentration of cinnamaldehyde, thymol and carvacrol could be decreased from 200,400 and $400 \mathrm{mg} / \mathrm{L}$ to 100,100 and $100 \mathrm{mg} / \mathrm{L}$, respectively (Zhou et al., 2007). In this study we used cinnamaldehyde in its trans isomer, which is the form that is present as a major component of bark extract of cinnamon (Kollanoor Johny et al., 2008).

The MIC method has previously been used to evaluate the effectiveness of orange EOs against different Salmonella serovars (Enteritidis, Seftenberg, Tennessee, Kentucky, Heidelberg, Montevideo, Michigan, Stanley, among others) (O'Bryan, Crandall, Chalova, \& Ricke, 2008). These authors reported that three out of the seven citrus EOs evaluated (orange terpenes, d-limonene, and terpenes purified fractions from orange essence) exhibited antimicrobial activity, and that there was not a significant difference in the responses among the Salmonella serovars evaluated (O'Bryan et al., 2008). However, orange terpenes and d-limonene exhibited the most antimicrobial activity with a MIC of $1 \%$ for each serovar, and terpenes from orange essence exhibited MIC range from 0.125 to $0.5 \%$ (O'Bryan et al., 2008). In the current study, no effect was observed for any of the $S$. Heidelberg isolates subjected to orange terpenes. These contrasting observations may be due to the fact that O'Bryan et al. (2008) evaluated commercial essential oils and fractions (single fold d-limonene), which may contain additional antimicrobial properties that were not present in the essential oils evaluated in the current study. Additionally, despite the fact that limonene is one of the more well-known and characterized of the EOs from citrus products (Dabbah et al., 1970; Caccioni, Guizzardi, Biondi, Renda, \& Ruberto, 1998) which can exert potent, broad-spectrum antimicrobial activity (Di Pasqua, Hoskins, Betts, \& Mauriello, 2006), this essential oil, in the form of $\mathrm{R}(+)$-limonene did not exhibit any antimicrobial activity in this study.

Essential oils have also been studied to have antimicrobial activity against Salmonella when used post-harvest. In their research, Alali et al. (2013) determined the effect of non-pharmaceuticals (a blend of organic acids, a blend of EOs, lactic acids, and a combination of levulinic acid and sodium dodecyl sulfate) on weight gain, feed conversion ratio, mortality of broilers and their ability to reduce colonization and fecal shedding of $S$. Heidelberg. Their results showed that the broilers that received the EOs had significantly increased weight gain and mortalities were lower compared to other treatments. Salmonella Heidelberg contamination in crops was significantly lower in challenged and unchallenged broilers that received EOs and lactic acids in drinking water, when compared to other treatments (Alali et al., 2013). Results of our study further supports that some EOs could be used as antimicrobial agents against the foodborne pathogen Salmonella. Further studies will be needed to confirm the antimicrobial effect of these EOs in vivo and which combinations represent optimal antimicrobial activity.

Hoffmann et al. (2014) compared the genomes of 44 S. Heidelberg strains, and identified at least 273 genes that varied among these strains. Uniform responses to various EOs were observed for each $S$. Heidelberg isolate in this study, which suggests that the inhibitory mechanism(s) of EOs result from a broad inhibitory action such as potentially a membrane effect. Additionally, the genetic diversity among $S$. Heidelberg isolates may not play a role in the efficacy of survival when exposed to EOs. Additional studies on $S$. Heidelberg and EOs may be necessary to identify the most appropriate pre- and post-harvest applications of essential oil for limiting Salmonella spp. in the food supply.

\section{Acknowledgments}

We thank Dr. Steven L. Foley, Division of Microbiology, NCTR, U.S. Food and Drug Administration, Jefferson, AR, and Dr. W. Florian Fricke, University of Maryland, School of Medicine, Baltimore, MD for providing Salmonella Heidelberg strains used in this study, and Dr. Young M. Kwon, Poultry Science Department, University of Arkansas, Fayetteville, AR for providing the EOs for the study. We also thank the Department of Food Science, University of Arkansas, Fayetteville, AR for providing graduate stipend support to authors J. R. C. and C. A. B, as well as the Michael Johnson Scholarship to C.A.B.

\section{References}

Alali, W. Q., Hofacre, C. L., Mathis, G. F., \& Faltys, G. (2013). Effect of essential oil compound on shedding and colonization of Salmonella enterica serovar Heidelberg in broilers. Poultry Science, 92, 836-841. http://dx.doi.org/10.3382/ps.2012-02783

Alali, W. Q., Hofacre, C. L., Mathis, G. F., Faltys, G., Ricke, S. C., \& Doyle, M. P. (2013). Effect of non-pharmaceutical compounds on shedding and colonization of Salmonella enterica serovar Heidelberg in broilers. Food Control, 31, 125-128. http://dx.doi.org/10.1016/j.foodcont.2012.10.001 
Bajpai, V. K., Baek, K. H., \& Kang, S. C. (2012). Control of Salmonella in foods by using essential oils: a review. Food Research International, 45, 722-734. http://dx.doi.org/10.1016/j.foodres.2011.04.052

Bronowski, C., \& Winstanley, C. (2009). Identification and distribution of accessory genome DNA sequences from an invasive African isolate of Salmonella Heidelberg. FEMS Microbiology Letters, 298, 29-36. http://dx.doi.org/10.1111/j.1574-6968.2009.01697.x

Caccioni, D. R. L., Guizzardi, M., Biondi, D. M., Renda, A., \& Ruberto, G. (1998). Relationship between volatile components of citrus fruit essential oils and antimicrobial action on Penicillium digitatum and Penicillium italicum. International Journal of Food Microbiology, 43, 73-79. http://dx.doi.org/10.1016/S0168-1605(98)00099-3

CDC. (2006). Salmonella Surveillance: Annual Summary, 2006. Atlanta, Georgia US Department of Health and Human Services, CDC. 2008. Retrieved February 23, 2015, from http://198.246.124.22/ncidod/dbmd/phlisdata/salmtab/2006/SalmonellaIntroduction2006.pdf

Chaves, A. V., He, M. L. Yang, W. Z., Hristov, A. N., McAllister, T. A., \& Benchaar, C. (2008). Effects of essential oils on proteolytic, deaminative and methanogenic activities of mixed ruminal bacteria. Canadian Journal of Animal Science, 88, 117-122. http://dx.doi.org/10.4141/CJAS07061

Dabbah, R., Edwards, V. M., \& Moats, W.A. (1970). Antimicrobial action of some citrus fruit oils on selected food-borne bacteria. Applied Microbiology, 19, 27-31.

Di Pasqua, R., Hoskins, N., Betts, G., \& Mauriello, G. (2006). Changes in membrane fatty acids composition of microbial cells induced by addiction of thymol, carvacrol, limonene, cinnamaldehyde, and eugenol in the growing media. Journal of Agriculture and Food Chemistry, 54, 2745-2749. http://dx.doi.org/10.1021/jf0527221

FDA. (2009). National Antimicrobial Resistance Monitoring System-Enteric Bacteria (NARMS): 2006 Executive Report. U.S. Department of Health and Human Services, U.S. FDA. Accessed February 23, 2015. http://www.fda.gov/downloads/AnimalVeterinary/SafetyHealth/AntimicrobialResistance/NationalAntimicro bialResistanceMonitoringSystem/UCM312360.pdf

Fisher, K., \& Phillips, C. A. (2008). Potential antimicrobial uses of essential oils in food: is citrus the answer? Trends in Food Science and Technology, 19, 156-164. http://dx.doi.org/10.1016/j.tifs.2007.11.006

Foley, S. L., \& Lynne, A. M. (2008). Food animal-associated Salmonella challenges: pathogenicity and antimicrobial resistance. Journal of Animal Science, 86, E173-E187. http://dx.doi.org/10.2527/jas.2007-0447

Foley, S. L., Nayak, R. Hanning, I. B., Johnson, T. J., Han, J., \& Ricke, S. C. (2011). Minireview: Population dynamics of Salmonella enterica serotypes in commercial egg and poultry production. Applied and Environmental Microbiology, 77, 4273-4279. http://dx.doi.org/10.1128/AEM.00598-11

Fricke, W. F., Mammel, M. K., McDermott, P. F., Tartera, C., White, D. G., LeClerc, J. E., ... Cebula, T. A. (2011). Comparative genomics of 28 Salmonella enterica isolates: evidence for CRISPR-mediated adaptive sublineage evolution. Journal of Bacteriology, 193, 3556-3568. http://dx.doi.org/10.1128/JB.00297-11

González-Gil, F., Le Bolloch, A., Pendleton, S., Zhang, N., Wallis, A., \& Hanning, I. (2012). Expression of hilA in response to mild acid stress in Salmonella enterica is serovar and strain dependent. Journal of Food Science, 77, M292-M296. http://dx.doi.org/10.1111/j.1750-3841.2012.02684.x

Han, J., David, D. E., Deck, J., Lynne, A. M., Kaldhone, P., Nayak, R., ... Foley, S. L. (2011). Comparison of Salmonella enterica serovar Heidelberg isolates from human patients with those from animal and food sources. Journal of Clinical Microbiology, 49, 1130-1133. http://dx.doi.org/10.1128/JCM.01931-10

Han, J., Lynne, A. M., David, D. E., Tang, H. E., Xu, J., Nayak, R., ... Foley, S. L. (2012). DNA sequence analysis of plasmids from multidrug resistant Salmonella enterica serotype Heidelerg isolates. PLoS One, 7, e51160. http://dx.doi.org/10.1371/journal.pone.0051160

Heithoff, D. M., Shimp, W. R., House, J. K., Xie, Y., Weimer, B. C., Sinsheimer, R. L., \& Mahan, M. J. (2012). Intraspecies variation in the emergence of hyperinfectious bacterial strains in nature. PLoS Pathogens, 8, 1-17. http://dx.doi.org/10.1371/journal.ppat.1002647

Hoffmann, M., Zhao, S., Pettengill, J., Luo, Y., Monday, S. R., Abbot, J., ... McDermott, P. F. (2014). Comparative genomic analysis and virulence differences in closely related Salmonella enterica serotype Heidelberg isolates from humans, retail meats, and animals. Genome Biology and Evolution, 6, 1046-1068. 
http://dx.doi.org/10.1093/gbe/evu079

Hur, J., Jawale, C., \& Lee, J. H. (2012). Antimicrobial resistance of Salmonella isolated from food animals: A review. Food Research International, 45, 819-830. http://dx.doi.org/10.1016/j.foodres.2011.05.014

Kaldhone, P., Nayak, R., Lynne, A. M., David, D. E., McDermott, P. F., Logue, C. M., \& Foley, S. L. (2008). Characterization of Salmonella enterica serovar Heidelberg from turkey-associated sources. Applied and Environmental Microbiology, 74, 5038-5046. http://dx.doi.org/10.1128/AEM.00409-08

Kim, W. K., Karabasil, N., Bulajic, S., Dunkley, K. D., Callaway, T. R. Poole, T. L., ... Nisbet, D. J. (2005). Comparison of spontaneous antibiotic resistance frequency of Salmonella Typhimurium growth in glucose amended continuous culture at slow and fast dilution rates. Journal of Environmental Science and Health Part B, 40, 475-484. http://dx.doi.org/10.1081/PFC-200047598

Kollanoor Johny, Darre, A. M. J., Hoagland, T. A., Schreiber, D. T., Donoghue, A. M., Donoghue, D. J., \& Venkitanarayanan, K. (2008). Antibacterial effect of trans-cinnamaldehyde on Salmonella Enteritidis and Campylobacter jejuni in chicken drinking water. Journal of Applied Poultry Research, 17, 490-497. http://dx.doi.org/10.3382/japr.2008-00051

Logue, C. M., Sherwood, J. S. Olah, P. A., Elijah, L. M., \& Dockter, M. R. (2003). The incidence of antimicrobial-resistant Salmonella spp. on freshly processed poultry from US Midwestern processing plants. Journal of Applied Microbiology, 94, 16-24. http://dx.doi.org/10.1046/j.1365-2672.2003.01815.x

Luo, Y., Kong, Q., Yang, J., Mitra, A., Golden, G., Wanda, S. Y., ... Curtis III, R. (2012). Comparative genome analysis of the high pathogenicity Salmonella Typhimurium strain UK-1. PLoS One, 7, e40645. http://dx.doi.org/10.1371/journal.pone.0040645

Lynne A. M., Kaldhone, P., David, D., White, D. G., \& Foley, S. L. (2009). Characterization of antimicrobial resistance in Salmonella enterica serotype Heidelberg isolated from food animals. Foodborne Pathogens and Diseases, 6, 207-215. http://dx.doi.org/10.1089/fpd.2008.0172

Nayak, R., Stewart, T., Wang, R. F., Lin, J., Cerniglia, C. E., \& Kenney, P. B. (2004). Genetic diversity and virulence gene determinants of antibiotic-resistant Salmonella isolated from preharvest turkey production sources. International Journal of Food Microbiology, 91, 51-62. http://dx.doi.org/10.1016/S0168-1605(03)00330-1

O’Bryan, C. A., Crandall, P. G., Chalova, V. I., \& Ricke, S. C. (2008). Orange essential oils antimicrobial activities against Salmonella spp. Journal of Food Science, 73, M264-M267. http://dx.doi.org/10.1111/j.1750-3841.2008.00790.x

Oloya, J., Doetkott, D., \& Khaitsa, M. L. (2009). Antimicrobial drug resistance and molecular characterization of Salmonella isolated from domestic animals, humans, and meat products. Foodborne Pathogens and Disease, 6, 273-284. http://dx.doi.org/10.1089/fpd.2008.0134

Park, S. H., \& Ricke, S. C. (2015). Development of multiplex PCR assay for simultaneous detection of Salmonella genus, Salmonella subspecies I, Salm. Enteritidis, Salm. Heidelberg and Salm. Typhimurium. Journal of Applied Microbiology, 118, 152-160. http://dx.doi.org/10.1111/jam.12678

Ravishankar, S., Zhu, L., Reyna-Granados, J., Law, B., Joens, L., \& Friedman, M. (2010). Carvacrol and cinnamaldehyde inactivate antibiotic-resistant Salmonella enterica in buffer and on celery and oysters. Journal of Food Protection, 73, 234-240.

Rivera Calo, J., Crandall, P. G., O’Bryan, C. A., \& Ricke, S. C. (2015a). Essential oils as antimicrobials in food systems - A review. Food Control, 54, 111-119. http://dx.doi.org/10.1016/j.foodcont.2014.12.040

Rivera Calo, J., Park, S. H., Baker, C. A., \& Ricke, S. C. (2015b). Specificity of Salmonella Typhimurium strain (ATCC 14028) growth responses to Salmonella serovar-generated spent media. Journal of Environmental Science and Health Part B, 50, 422-429. http://dx.doi.org/10.1080/03601234.2015.1011962

Scallan, E., Hoekstra, R. M., Angulo, F. J., Tauxe, R. V., Widdowson, M-A., Roy, S. L., ... Griffin, P. M. (2011). Foodborne illness acquired in the United States-major pathogens. Emerging Infectious Diseases, 17, 7-15. http://dx.doi.org/10.3201/eid1707.110572

Scharff, R. L. (2010). Health-related costs from foodborne illness in the United States. Retrieved February 28, 2015 , from http://publichealth.lacounty.gov/eh/docs/ReportPublication/HlthRelatedCostsFromFoodborneIllinessUS.pdf

Shah, D. H., Zhou, X., Addwebi, T., Davis, M. A., Orfe, L., Call, D. R., ... Besser, T. E. (2011). Cell invasion of 
poultry-associated Salmonella enterica serovar Enteritidis isolates is associated with pathogenicity, motility and proteins secreted by the type III secretion system. Microbiology, 157, 1428-1445. http://dx.doi.org/10.1099/mic.0.044461-0

Sivropoulou, A., Papanikolaou, E. Nikolaou, C. Kokkini, S. Lanaras, T., \& Arsenakis, M. (1996). Antimicrobial and cytotoxic activities of Origanum essential oils. Journal of Agricultural and Food Chemistry, 44, 1202-1205. http://dx.doi.org/10.1021/jf950540t

Solórzano-Santos, F., \& Miranda-Novales, M. G. (2012). Essential oils from aromatic herbs as antimicrobial agents. Current Opinions in Biotechnology, 23, 136-141. http://dx.doi.org/10.1016/j.copbio.2011.08.005

Su, L.-H., Chiu, C.-H., Chu, C., \& Ou. J. T. (2004). Antimicrobial resistance in nontyphoid Salmonella serotypes: a global challenge. Clinical Infectious Diseases, 39, 546-551. http://dx.doi.org/10.1086/422726

Vugia, D. J., Samuel, M., Farley, M. M., Marcus, R., Shiferaw, B., Shallow, S., Smith, K., \& Angulo, F. J. (2004). Invasive Salmonella infections in the United States, FoodNet, 1996-1999: incidence, serotype distribution, and outcome. Clinical Infectious Diseases, 38, S149-156. http://dx.doi.org/10.1086/381581

Watson, P. R., Paulin, S. M., Bland, A. P., Jones, P. W., \& Wallis, P. S. (1995). Characterization of intestinal invasion by Salmonella typhimurium and Salmonella dublin and effect of a mutation in the invH gene. Infection and Immunity, 63, 2743-2754.

Wilmshurst, P., \& Sutcliffe, H. (1995). Splenic abscess due to Salmonella Heidelberg. Clinical Infectious Diseases, 21, 1065-1066. http://dx.doi.org/10.1093/clinids/21.4.1065

Zhao, S., White, D. G., Friedman, S. L., Glenn, A., Blickenstaff, K., Ayers, K. L., ... McDermott, P. F. (2008). Antimicrobial resistance in Salmonella enterica serovar Heidelberg isolates from retail meats, including poultry, from 2002 to 2006. Applied and Environmental Microbiology, 74, 6656-6662. http://dx.doi.org/10.1128/AEM.01249-08

Zhou, F., Ji, B., Zhang, H., Jiang, H., Yang, Z., Li, J., \& Yan, W. (2007). The antibacterial effect of cinnamaldehyde, thymol, carvacrol and their combinations against the foodborne pathogen Salmonella Typhimurium. Journal of Food Safety, 27, 124-133. http://dx.doi.org/10.1111/j.1745-4565.2007.00064.x

\section{Copyrights}

Copyright for this article is retained by the author(s), with first publication rights granted to the journal.

This is an open-access article distributed under the terms and conditions of the Creative Commons Attribution license (http://creativecommons.org/licenses/by/3.0/). 